\title{
Anomalous Size Dependent Rheological Behavior of Alumina Based Nanofluids
}

\author{
Pawan K. SINGH ${ }^{1}$, Anoop K. B ${ }^{1}$, H. E. PATEL ${ }^{1}$, T. SUNDARARAJAN ${ }^{1}$, T.PRADEEP ${ }^{2}$, Sarit. K.DAS ${ }^{1, *}$ \\ * Corresponding author: Tel.: ++91 (44)22574655; Fax: ++91 (44)22570545; Email: skdas@iitm.ac.in \\ 1: Department of Mechanical Engineering, Indian Institute of Technology Madras, India \\ 2: Department of Chemistry and SAIF, Indian Institute of Technology Madras, India
}

\begin{abstract}
Rheological behaviour of Alumina $\left(\mathrm{Al}_{2} \mathrm{O}_{3}\right)$ based nanofluids (NFs) has been studied and found to be exhibit unexpected behaviour. Two base-fluids viz, water and ethylene glycols (EG). Three particle sizes (11, 45 and $150 \mathrm{~nm}$ ), varying over an order of magnitude, were used to analyze the effect of particle size. The experimental data has shown typical Newtonian behavior for both W based and EG based alumina NFs The viscosity of EG based NFs is found to be anomalously reduced compared to the base fluid. This reduction in viscosity may be due to hygroscopic nature of EG or due to the presence of water in as-received high concentration sample also, as told by some researchers. However, this phenomenon was absent for water based NFs. The inter-related effects of particle size, concentration and mode of dispersion (mono or poly-dispersed) were investigated. To eliminate the effect of size variation, mono dispersed NFs are obtained by centrifuging and re-suspension of parent NFs. Particle migration under shear is attributed to the reduction of viscosity. The increase in bulk viscosity with particle size reduction is attributed to the surface forces acting between the particles and the medium in a suspension.
\end{abstract}

Keywords: Nanofluids, alumina suspensions, shear induced particle migration, rheology of suspensions, viscosity of suspensions

\section{Introduction}

Due to increasing demand of electronic cooling industry, it asks for better heat transfer methods. It can be increased in two ways, first by changing the geometry and second by using the better cooling fluids. NFs (Nanofluids) can be a option due to its better heat transfer characteristics. As the definition given by Das (2008), Nanofluids are the engineered dilute colloidal suspension of nano sized (less than $100 \mathrm{~nm}$ ) particles in base fluids. The thermal conductivity of nanofluids has been studied by many researchers in past [Lee et al.[1999], Choi et al.[2001], Patel et al.[2003] Das et al.[2003]] and they showed anomalous heat transfer and thermal conductivity behaviour of nanofluids. However the usage of these fluids needs the comprehensive study of its thermophysical property and effective viscosity is one of these properties which govern the ease of flow and pressure drop during the flow. The early viscosity studies of alumina-water nanofluids is done by Pak and Cho[1998] and they showed that the viscosity values are considerably larger than that of the classical theory of dilute suspension, which is represented by Einstein[1911]. Putra et al.(2003) demonstrated the Newtonian behaviour and temperature effect of aluminawater nanofluids. In similar studies of alumina-water by Wang and Kurma [2008] the effect of temperature and concentration has been showed. And it was found that effective viscosity increase with concentration and decrease with temperature. They used these results to explain the thermal conductivity behaviour. To see the effect of base fluid other than water, rheological data on alumina based NFs with varying diameters (27, 40 and 50 $\mathrm{nm})$ suspended in propyleneglycol were reported by Prasher et al.[2006]. The viscosity ratio was largely independent of particle sizes and the aggregation of particles in NFs was indicated to be the cause of under-prediction by Einstein's equation. Lee et al.[2008] studied the behaviour of low concentrations alumina- water nanofluids and found a non linear behaviour of viscosity against concentration. 
These all literature suggests that the viscosity studies of nanofluids are still sparse. Also many investigators did not consider that the in a nanofluids the particle size distribution is very large, which can affects physical behaviour of nanofluids at these nanolevels.

Present article aims to fill some gap in viscosity studies and reports some surprising observation on the effective viscosity of NFs while trying to investigate the effect of various parameters such as particle size, volume fraction, mode of dispersion and nature of base-fluids.

\section{Experimental methods}

Nanoparticles used in all the experiments are commercially purchased alumina nano-powder (Nanophase Technologies Corporation and Inframat Advanced Materials) and produced by physical methods. The nano-particles with the three particle size (11, 45 and $150 \mathrm{~nm})$, varying over an order of magnitude, were used to analyze the effect of particle size The two base-fluids used are water (W) and ethylene glycol (EG) to see the effect of different fluids. To prepare the nanofluids, the particles were dispersed in the base-fluids using several hours of ultrasonication and no stabilizers were used as they may change the rheological properties. Figure 1(a)-(c) show the TEM images of typical $\mathrm{Al}_{2} \mathrm{O}_{3}$ particles in the 'as received' state. The average particle dimensions were measured by gas absorption method and were 11, 45 and $150 \mathrm{~nm}$.

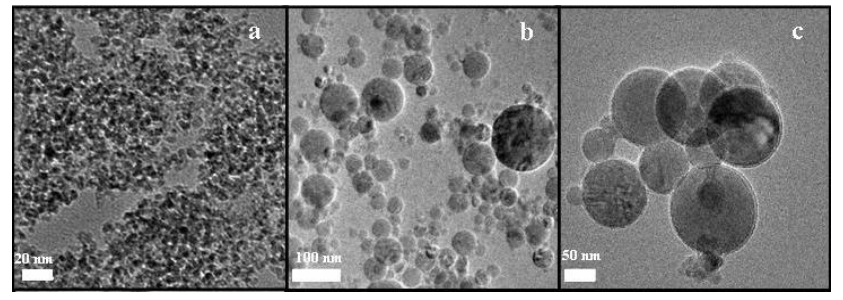

Figure1: TEM of particles used in the preparation of NFs. 'As received'

a) 11 , b) 45 and c) $150 \mathrm{~nm}$

For measurement of rheological properties of Ethylene glycol (EG) based nanofluids, a rheometer of Anton Paar make (Physica MCR 301 ) with cone and plate geometry (50 mm diameter $2^{0}$ angle with $47 \mu \mathrm{m}$ gap) is used.
The temperature of plate is controlled by a separate Peltier unit. The measurement was at the rate of 50 readings in 10 second interval and the shear rate was varied from $50 \mathrm{~s}^{-1}$ to $250 \mathrm{~s}^{-1}$. The relative viscosities of water based NFs were measured using an Ubbelohde viscometer as the viscosity values were smaller for applying the rheometer with sufficient accuracy. The above comparative viscometer had a capillary bore of $0.5 \mathrm{~mm}$. The ratio of efflux time of NF, $t_{\text {nano }}$ to that of base-fluid $t_{b f}$ was taken as the experimental relative viscosity ratio.

i.e,

$$
\eta_{r}=\frac{t_{\text {nano }}}{t_{b f}}
$$

The inaccuracy involved in the determination of viscosity ratio was in the range of 0.5 to $1 \%$.

As visible from TEMs, the particle size in a sample can vary over a range, e,g. in a $11 \mathrm{~nm}$ particle size sample, the particle size may vary from $3 \mathrm{~nm}$ to $50 \mathrm{~nm}$ ). Hence the suspension obtained with these particles was polydispersed and they can not truly represent a fixed size of nanoparticles. To obtain more uniform size variation, the parent suspension was centrifuged successively. For example first, a NF solution of 1 vol\% concentration was prepared by mixing the required amount of $45 \mathrm{~nm} \mathrm{Al} \mathrm{Al}_{3}$ nanoparticles with water as the base fluid and sonicating for 5-6 hrs. This solution was first centrifuged at $1200 \mathrm{rpm}$. The residue was extracted. The mother liquor was centrifuged again at $2200 \mathrm{rpm}$ and the residue was extracted. The centrifugate was further centrifuged at $3200 \mathrm{rpm}$ to extract a residue. The residue obtained at three different centrifugation speeds corresponded to three narrower size distributions, collected from an average particle size. The TEM images of 45 $\mathrm{nm} \mathrm{Al}_{2} \mathrm{O}_{3}$ nanoparticles after centrifugation at the three speeds are shown in Figure 1(d)-(f), which clearly suggest that the particles prepared are more uniform in size. These particles are labeled as 45-1200, 45-2200 and 45-3200, respectively in the subsequent description. In $150 \mathrm{~nm}$ average size, different centrifugation speeds were employed.

After drying these particles, the NFs of 0.5, 
0.25 and 0.125 vol\% were prepared by mixing the required amount of nanoparticles in EG and sonicating the mixture for 5-6 hrs. Again no stabilizer was used. Similarly other samples were also prepared from $11 \mathrm{~nm}$ and $150 \mathrm{~nm}$ nanoparticles. 50 readings in the shear rate range, $50 \mathrm{~s}^{-1}$ to $250 \mathrm{~s}^{-1}$ were taken for these nearly monodispersed NFs.

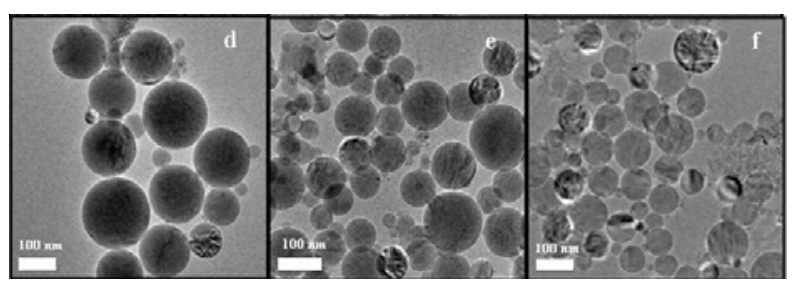

Figure 2: TEM of centrifuged $45 \mathrm{~nm}$ (average size) particles at speeds of d) 1200 , e) 2200 and f) $3200 \mathrm{rpm}$.

For measurement of thermal conductivity of NFs, the transient hot wire instrument developed by Patel et al.(2003) was used.

\section{Results and Discussions}

Figure 3 shows the rheological behavior of EG based polydispersed 1 vol\% alumina NFs. The linear dependence of shear stress to strain rate shows the Newtonian nature of the NF in the shear rate range $50 \mathrm{~s}^{-1}$ to $250 \mathrm{~s}^{-1}$. Figure 2(b) shows that the relative viscosity ratio for $\mathrm{Al}_{2} \mathrm{O}_{3}$-EG polydispersed $\mathrm{NF}$ (2 vol\%) is independent of strain rate in the range of $50 \mathrm{~s}^{-1}$

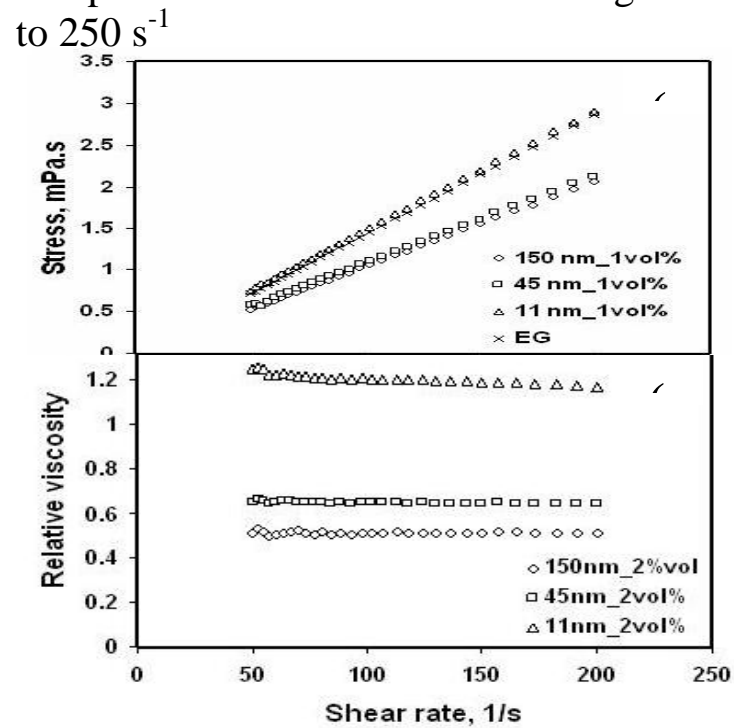

Figure 3: Rheological behavior of EG based polydispersed alumina NFs:

(a) shear stress versus strain rate

(b) viscosity versus shear rate.
It may be also noted that for NFs with average particle diameters of 150 and $45 \mathrm{~nm}$, the relative viscosity has reduced below unity, but not for $11 \mathrm{~nm}$ nanoparticles. This is a unique and anomalous phenomenon since with the addition of particles; the suspension viscosity is expected to increase generally. Also as the particle size reduces, the bulk viscosity increases. To confirm this unique behavior and its size sensitivity, similar curves were drawn for nearly monodispersed NFs derived from 45 $\mathrm{nm}$ nanoparticles (Fig.4).

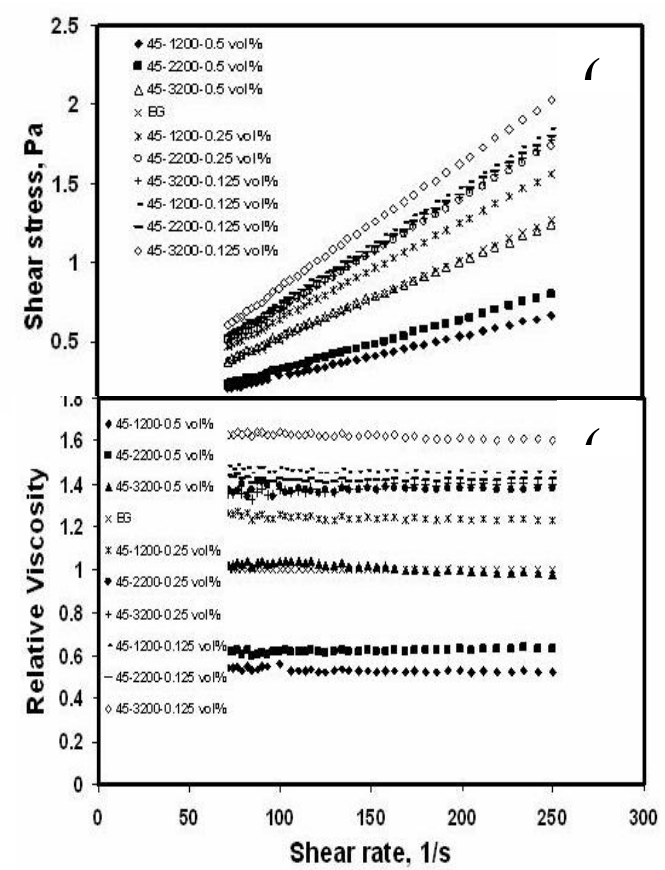

Figure 4: Rheological behavior of EG based nearly monodispersed NFs derived from $45 \mathrm{~nm}$ particles: (a)shear stress versus strain rate and (b) viscosity versus shear rate.

The relative viscosity for 0.5 vol\% is below unity for all samples 45-1200, 45-2200 and 453200 , but not for lower concentrations of 0.25 vol\% and 0.125 vol\%. It shows that this unique behavior occurs only at certain combinations of particle size and concentration. It also suggests that there are threshold concentrations at both ends (higher and lower concentration side) for getting reduced viscosity. For $11 \mathrm{~nm}$ and $150 \mathrm{~nm}$ (not shown here) particles, similar behavior is observed. For $150 \mathrm{~nm}$ particles, reduced viscosity is not visible. 
However, this phenomenon was observed for only EG based NFs and was absent for water based NFs. In this case, the viscosity increased in all the cases, as expected. The viscosity ratio variation for polydispersed NFs is plotted in Figure 5 at a constant strain of $150 \mathrm{~s}^{-1}$ for different particle sizes.

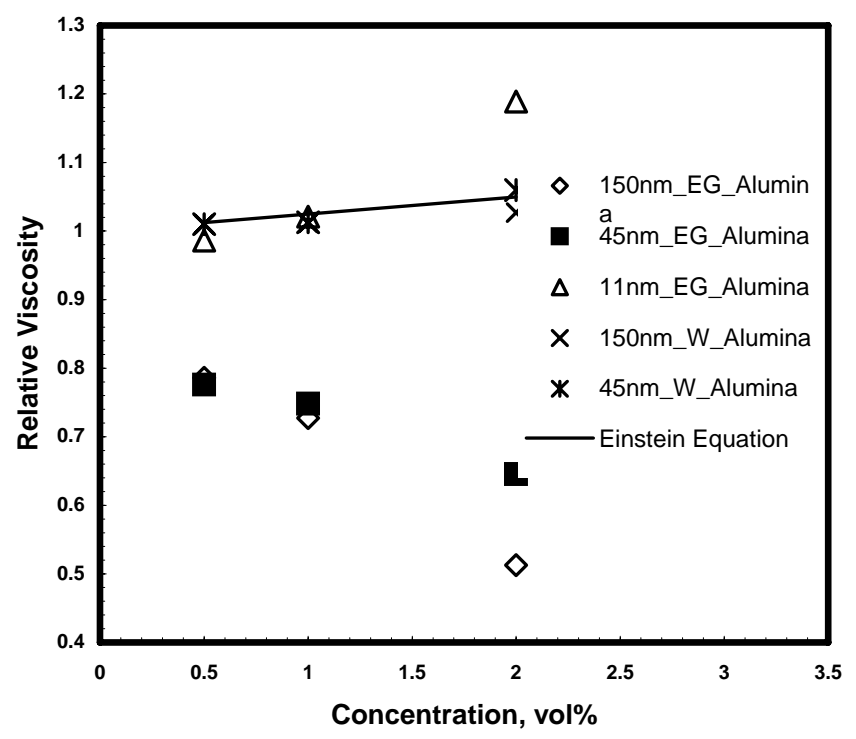

Figure 5: Variation of relative viscosity with concentration for water and EG based poly dispersed NFs

For EG based NFs, with $150 \mathrm{~nm}$ and $45 \mathrm{~nm}$ particles, the viscosity ratio reduces with particle concentration while for NFs with 11 $\mathrm{nm}$ particles, the ratio increases. Theoretical prediction using Einstein's equation is also plotted for comparison. This figure also shows the variation for $\mathrm{Al}_{2} \mathrm{O}_{3}$-water NF. In almost all cases with water as the based fluid, the viscosity ratio is above unity and the effect of concentration on viscosity of NFs made with $11 \mathrm{~nm}$ particle is more significant. Einstein's theory predicts the viscosity of 150 and $45 \mathrm{~nm}$ water based NFs more or less reasonably while the prediction for $11 \mathrm{~nm}$ deviates considerably. The above result shows that the particle diameter, concentration and nature of the base fluid have profound influence on NF viscosity and deviates considerably from conventional theory, and this phenomenon has not been reported in literature.

Two major observations are made from the experimental results. Firstly, viscosity of EG based alumina NF shows anomalously reduced viscosity compared to the base fluid, while it was not observed for water based NFs. A similar behavior of Alumina-EG NFs (reduced viscosity behaviour) was also observed by Jermey B. Gorden (2007) at MIT in his MS study. He has attributed this strange behaviour to the presence of water in as-received high concentration sample, which is questionable, since the reduced viscosity behaviour occurs at some particular sizes and concentration only. Secondly observation was that with the particle size reduction, the viscosity increases and in the nanometer range, it starts deviating from the conventional theory. In usual cases, dilute suspensions display higher viscosity than the base fluid and follows Einstein's equation for suspensions (1911),

$$
\mu_{s}=\mu_{b f}(1+2.5 \phi)
$$

where $\mu_{\mathrm{s}}$ stands for viscosity of the suspension, $\mu_{\mathrm{bf}}$ for viscosity of base fluid and $\phi$ for volume fraction. Any deviation from the above requires an explanation.

Particle migration in NFs has been reported in detail by Buongiorno (2006) and Ding and Wen (2005). In the present study, the reason for reduction of viscosity ratio for ethylene glycol based NFs is likely to be due to shear induced particle migration. Particle migration of Brownian suspensions under shear was shown experimentally by Frank et al.(2003) using confocal microscopy. According to Ding(2005), due to this shear induced particle migration, viscosity may be non uniform across the transverse plane, but if we see the overall measured viscosity of some particular nanofluids, it is reduced. Also one may argue that this reduced viscosity behavior is due to slip taking place near the walls through the sticking nanoparticles to the walls. If this is true, viscosity should reduce for all sizes, fluids and concentrations. But this behavior is seen only at some particular combinations of fluid, particle size and concentration with perfect repeatability. Hence, definitely any sweeping reason such as instrument error or particle slip is not the reason behind this strange behavior. Therefore the only probable reason left is in the physics of particle-fluidsurface interaction such as shear induced 
particle migration, which needs to be confirmed by theoretical estimation of the order of various forces at different ranges of particle sizes, fluids and concentrations, which is presented below.

A non-dimensional Peclet number is conceptually a ratio of time required for Brownian diffusion to move a particle to the time required for shear flow to move the same distance and can be used as a parameter for determining the influence of shear. Thus Peclet number $(\mathrm{Pe})$ may be defined as

$$
P e=\frac{6 \pi \eta_{0} \gamma a^{8}}{k T}
$$

Where $a$ is the particle radius, $\eta_{0}$ is the basefluid viscosity, $\gamma$ is the strain rate, $k$ the Boltzmann constant and $\mathrm{T}$ is the absolute temperature.

Figure 6 shows the variation of $\mathrm{Pe}$ with particle size for two base fluids at a constant strain rate of $200 \mathrm{~s}^{-1}$.

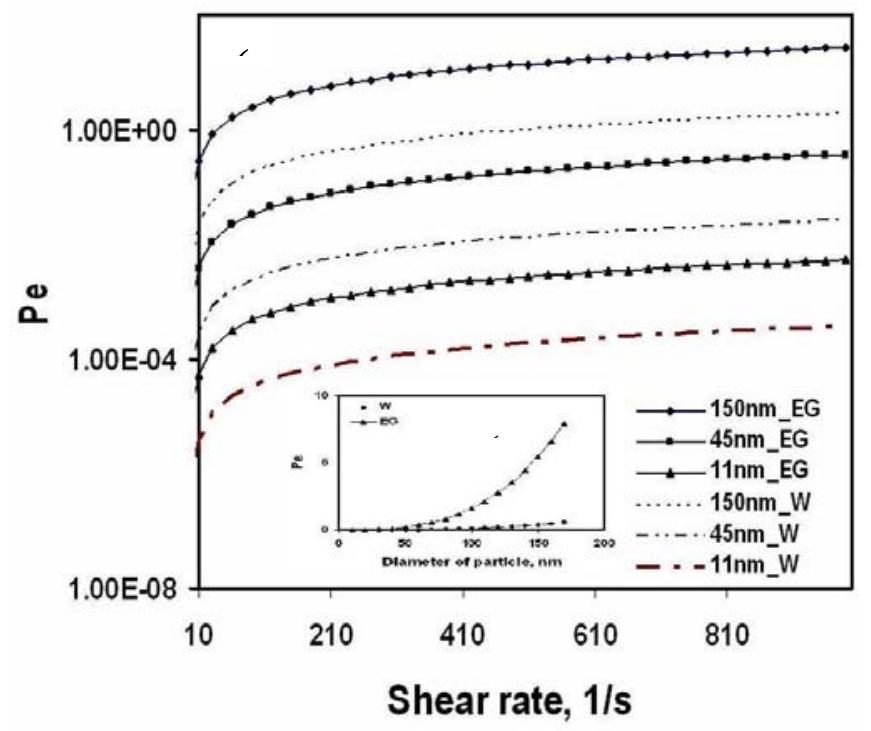

Figure 6: Pe variation with shear rates for different nanofluids. The variation with particle sizes is in the inset.

It can be observed that the values of $\mathrm{Pe}$ increases with particle size and are one to two orders higher for EG based NFs when compared with that of water based NFs. It clearly indicates that shear induce particle migration is higher for EG based NFs compared to that of water based ones. Figure 7(inset) shows variation of Pe with shear rate for different particle sizes, both for water and EG based NFs.

These observation helpe to expain the reduced viscosity behavious. It may be perceived here that within the interplay of repulsive forces and shear forces for suspensions with $11 \mathrm{~nm}$ particles, the repulsive forces dominate over the shear thus showing a relative viscosity above unity. In the case of EG based NFs, the particle migration under shear force helps as a lubricating effect, reducing the relative viscosity. The story is, however, different with water based NFs due to the polar nature of water. Due to this, van der Waals forces and the electric double layer dominate which reduce the particle migration under shear and induce the fluid to behave in the expected way. For using these NFs for heat transfer applications, it is sensible to compare the effect of thermal conductivity along with viscosity. It was shown by Prasher et al. (2006) that for NFs to be advantageous, the ratio of viscosity enhancement coefficient, $C_{\mu}$ to thermal conductivity enhancement coefficient, $C_{k}$ should be less than four. A comparison of viscosity ratio with conductivity ratio for water based alumina NFs for varying concentrations (Fig. 7) reveals that this is indeed the case.

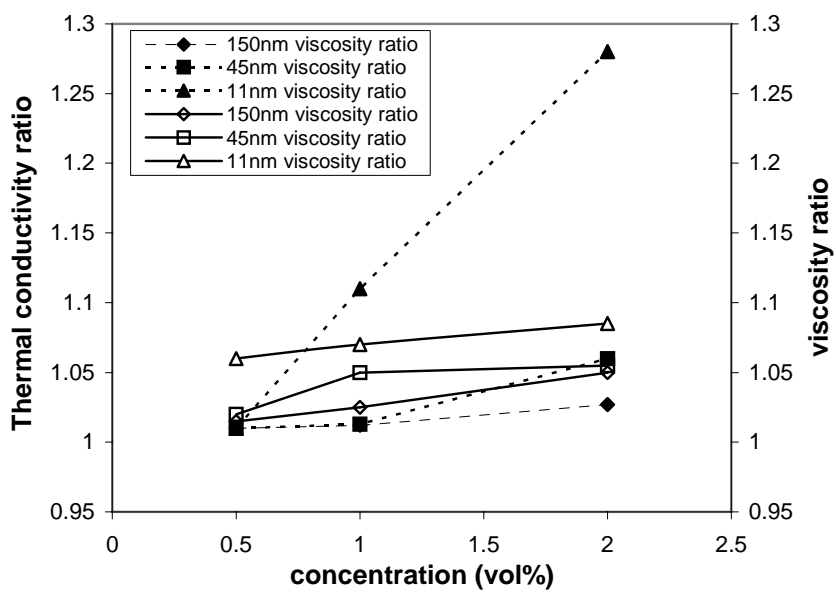

Figure 7: Variation of thermal conductivity ratio and viscosity ratio for water based Alumina NFs

It may thus be mentioned here that even though the viscosity has increased with 
reduction in particle sizes, the thermal conductivity values also increase proportionally, thus making it a prominent candidate as a cooling fluid. On the other hand, EG based alumina nanofluids are favorable for heat transfer applications, due to observed behavior of reduced viscosity and increased thermal conductivity.

\section{Conclusions}

In conclusion, the experimental data has shown typical Newtonian behavior for both $\mathrm{W}$ based and EG based alumina NFs. For the latter, for higher particle sizes a unique phenomenon of reduction in viscosity is observed in polydispersed NFs at certain range of concentration. These results and trends have been further confirmed by NFs obtained by centrifugation and resuspension of particles so that nearly monodispersed NFs are obtained, thus eliminating the effect of size variation. This phenomenon was found to be absent in the water based NFs. Analysis of these results attributes this phenomenon to particle migration under shear. It is also observed that as the particle size reduces the bulk viscosity values get increased, which is mainly due to the increase in surface attractive forces. Also the viscosities decrease with temperature and comparison with increase of thermal conductivity reveals that even with the increase in viscosity, NFs remain attractive candidates for next generation cooling.

\section{Acknowledgement}

The authors acknowledge the financial support of Department of Science and Technology, India. They would also like to acknowledge Prof. Abhijit Deshpande, Ms. Harini, Mrs. S. Banupriya, Mr. Anbu for their assistance and advice during the experiments.

\section{References:}

1. Buongiorno,J., 2006, Convective Transport in Nanofluids, J. Heat Tran., 128, 240-250.

2. Das, S. K.; Choi, S. U. S.; Yu, W., T. Pradeep, 2008, Nanofluids Science and Technology, John Wiley, New York
3. Das, S.K., Putra, N., Thiesen, P., Roetzel, W., 2003, Temperature dependence of thermal conductivity enhancement for nanofluids, J. Heat Transfer, Vol. 125 pp.567-74.

4. Ding, Y. L. and Wen, D. S, 2004, Particle migration in a flow of a suspension of nanoparticles (nanofluids), Powder Technology, 149, 84-92.

5. Einstein,A., 1911, Ann. Phys , 34, 591.

6. Frank, M.; Anderson, D.; Weeks, E. R.; Morris,J. F., 2003, Particle migration in pressure-driven flow of a Brownian suspension, J. Fluid Mech., 493, 363378.

7. Gordon, Jeremy B., 2007, Thermorheological Properties of Nanostructured Dispersions, MS Thesis (MIT 2007)

8. Lee J.H., Hwang K. S., Jang S. P., Lee B. H., Kim J.H., S.U.S. Choi, Chul Jin Choi, 2008, Effective viscosities and thermal conductivities of aqueous nanofluids containing low volume concentrations of $\mathrm{Al}_{2} \mathrm{O}_{3}$ nanoparticles, Int J. Heat Mass Transfer., 51, 26512656.

9. Nguyen, C.T.; Desgranges, F.; Galanis, N.; Roy, G.; Mare, T.; Boucher, S.; Mintsa, H.A., 2008, Viscosity data for $\mathrm{Al}_{2} \mathrm{O}_{3}$-water nanofluid-hysteresis: is heat transfer enhancement using nanofluids reliable?, Int. J. Thermal Sciences, 47 (2), 103-111.

10. Pak, B.; Cho, Y. I., 1998, Hydrodynamic and heat transfer study of dispersed fluids with submicron metallic oxide particles, Experimental Heat Trans., 11, 151-170.

11. Patel, H.E.; Das, S. K.; Sundararajan, T.; Sreekumaran N. A.; George, B.; Pradeep T., 2003, Thermal conductivities of naked and monolayer protected metal nanoparticle based nanofluids: manifestation of anomalous enhancement and chemical effects, Appl. Phys. Lett., 83 (14), 2931-3. 
12. Prasher,R.; Song, D.; Wang, J.; Phelan, P., 2006, Measurements of nanofluid viscosity and its implications for thermal applications, Appl. Phys. Lett., 89, 133108.

13. Putra, N.; Roetzel, W.; Das, S. K., 2003, Natural convection of nano-fluids, Heat and Mass Trans., 39, 775-784.

14. Ronald G. Larson, 1999, The structure and rheology of complex fluids (Chapter 6), Oxford university press, New York.

15. S. Lee, S.U.S Choi, S. Li, and J. A. Eastman, 1999, Measuring thermal conductivity of fluids containing oxide nanoparticles, J. Heat Trans., 121, 280289.

16. Verwey, E. J. W.; Overbeek, J. Th. G., Theory of the Stability of Lyophobic Colloids (Elsevier, Amsterdam, 1948).

17. Wong K.F.V., Kurma. T., Nanotechnology 19 (2008) 345702

18. Zhou, G. V. Z.; Duin, N. J.; Boger, D.V., 2000, Effect of interparticle forces on shear thickening of oxide suspensions, J. Rheol, 44, 759-779. 